\title{
Downregulation of Rab25 activates Akt1 in head and neck squamous cell carcinoma
}

\author{
DIDEM SEVEN $^{1}$, SOYDAN DOGAN ${ }^{1}$, ERKAN KILIÇ ${ }^{2}$, \\ EMIN KARAMAN ${ }^{2}$, HIKMET KOSEOGLU ${ }^{1}$ and NUR BUYRU ${ }^{1}$ \\ Departments of ${ }^{1}$ Medical Biology; ${ }^{2}$ Otorhinolaryngology, Istanbul University, \\ Cerrahpasa Medical Faculty, Istanbul 34098, Turkey
}

Received September 30, 2014; Accepted June 11, 2015

DOI: $10.3892 / \mathrm{ol} .2015 .3433$

\begin{abstract}
Several studies have suggested that Ras-associated binding 25 protein (Rab25) is involved in the pathogenesis of human cancer. Although it has been demonstrated that the development of head and neck squamous cell carcinoma (HNSCC) is the result of an accumulation of multiple sequential genetic and epigenetic alterations in key genes with important functions in cell growth and the cell cycle, recent studies have indicated that HNSCC is a complex and heterogenous disease. To the best of our knowledge, there is no data regarding the regulation of the Rab25 gene at the mRNA or protein level in HNSCC. Furthermore, available data on Rab25 expression in other types of cancer are conflicting. The aim of the present study was to investigate whether Rab25 is involved in the development and/or progression of HNSCC, and to analyze the mechanisms underlying its effects in this type of cancer. The expression of Rab25 mRNA in HNSCC tissues and adjacent non-tumor tissue samples was measured using reverse transcription-quantitative polymerase chain reaction, while the level of the Rab25, Akt1 and phosphorylated-Akt1 proteins was measured using western blotting. Expression of Rab25 mRNA and protein was downregulated in $69.1 \%$ and $56.1 \%$ of tumor tissue samples, respectively. This downregulation was associated with an increase in p-Akt1 expression, in the absence of a change in total Aktl protein levels, in tumor tissues compared with normal tissues. The current findings suggest that Rab25 acts as a tumor suppressor in HNSCC.
\end{abstract}

Correspondence to: Professor Nur Buyru, Department of Medical Biology, Istanbul University, Cerrahpasa Medical Faculty, 53 Kocamustafapasa Street, Kocamustafapasa, Fatih, Istanbul 34098, Turkey

E-mail: nbuyru@yahoo.com

Key words: Ras-associated binding 25 protein, Akt1, head and neck squamous cell carcinoma, tumor suppression, gene expression

\section{Introduction}

Head and neck cancer (HNC) is the 6th most common type of cancer worldwide (1), and comprises cancers of the aerodigestive tract, including the lips, oral cavity, nasal cavity, paranasal sinuses, pharynx, larynx, oropharynx, hypopharynx, salivary glands and local lymph nodes (2). Approximately $90 \%$ of all HNCs are squamous cell carcinomas (HNSCCs), which arise from the mucosal lining in these regions (2). The 5-year survival rate for HNSCC is among the lowest of the most common types of cancer; having remained at $64-67 \%$ for the last ten years (3). Approximately $80-90 \%$ of cases of HNSCC are attributed to prolonged tobacco and/or alcohol use (2). However, only a small fraction of those who consume tobacco or alcohol develop this disease, suggesting that genetic factors are also important in its pathogenesis (4-6). Fundamental to the genetic basis of all cancers is the overexpression of oncogenes and/or silencing of tumor suppressor genes. Aberrations, such as deletions or mutations of these genes may lead to uncontrolled cell division and cancer development.

Ras-associated binding ( $\mathrm{Rab}$ )-GTPases are the largest subfamily of small GTPases, and are involved in the regulation of intracellular vesicle transport and protein trafficking (7-12). Rab proteins associate with distinct membrane compartments within cells and determine the specificity of vesicle trafficking pathways; the nidi for the assembly of multiprotein complexes (13). More than 60 mammalian Rab proteins have been identified to date, all of which exert their specific function by cycling between a GTP-bound active conformation and, following GTP hydrolysis, a GDP-bound inactive state (14).

Rab25 was identified in 1993 as a member of the Rab family. Rab25 is also known as Rab11c, due to its sequence homology with the Rab11a and Rab1lb proteins (15). However, it is expressed only in epithelial cells, in contrast to the ubiquitously expressed Rab1la and Rab11b $(15,16)$. Functional studies have demonstrated that the Rab11 subfamily is involved in transcytosis, endocytic sorting and transport across polarized epithelial cells, as well as in apical vesicle recycling $(17,18)$.

He et al (16) reported that the expression of 9 human GTPases is upregulated in liver cancer and that 6 of these were Rab proteins. This was the first data that demonstrated an association between Rab25 and epithelial cancer. Subsequently, overexpression of Rab25 has been shown to be associated with 
numerous types of human cancer (19-21). However, recent studies have identified two opposing roles for Rab25 in tumor progression. Several groups have suggested that Rab25 exerts a tumor promoting function, and have proposed that its overexpression is associated with an increase in tumor aggression and invasiveness (22-25). Overexpression of Rab25 has been reported in ovarian (22-24), prostate (26), invasive breast (27), colon (15) and liver cancer (16), in addition to transitional cell carcinoma of the bladder $(27,28)$ and Wilms' tumor $(29)$, which indicates that Rab25 acts as an oncogene in these situations. By contrast, a documented association between loss of Rab25 expression and increased tumor development, indicates that Rab25 may function as a tumor suppressor in a number of types of cancer. Loss of Rab25 expression has been detected in certain breast and colon cancer tissues, and in a novel breast cancer cell line, which was generated from human mammary epithelial cells $(30,31)$. More recently, Zhang et al (25) reported that Rab25 is upregulated in the majority of bladder tumors and that this upregulation is associated with tumor invasion and metastasis. The in vitro studies by that group have also demonstrated that Rab25 exerts its oncogenic effect

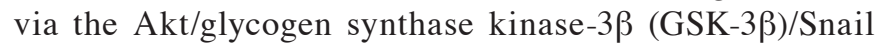
pathway. These conflicting results indicate that Rab25 may exert different effects in different types of tumor. To the best of our knowledge, only one study has investigated the role of Rab25 in HNC cell lines (32), the results of which suggested that Rab25 is involved in tumor migration and metastasis in HNSCC. The aim of the present study was to investigate the function of Rab25 in human HNSCC tissue samples and to examine its association with the Akt pathway. Therefore, the expression of Rab25 in HNSCC tumors was measured using reverse transcription-quantitative polymerase chain reaction (RT-qPCR) and western blotting.

\section{Materials and methods}

Tissue samples. Paired normal and tumor samples were obtained from 76 patients with HNSCC, who underwent surgery at the Department of Otorhinolaryngology (Cerrahpasa Medical Faculty, Istanbul, Turkey) following receipt of written informed consent. This study was approved by the Cerrahpasa Medical Faculty Ethics Committee (Approval no. 83045809/11295). Clinicopathological characteristics, including patient age, gender, TNM staging, histological grade, invasion status and tumor location, are presented in Table I.

$c D N A$ synthesis and RT-qPCR. Total RNA was extracted from tumor and adjacent non-tumor samples using the PureLink $^{\mathrm{TM}}$ RNA Mini kit (Ambion Life Technologies, Carlsbad, CA, USA). Single-stranded cDNA was synthesized using the Revert-Aid first strand cDNA synthesis kit (Fermantas, Vilnius, Lithuania) and $300 \mathrm{ng}$ of total RNA in a reaction volume of $20 \mu \mathrm{l}$. Glucose-6-phosphate dehydrogenase (G6PD) was used as a reference gene for normalization. The resultant cDNA $(2 \mu \mathrm{l})$ was used for the Real-Time assay, which was performed in $20 \mu \mathrm{l}$ reaction volume, containing $1 \mathrm{X}$ LightCycler 480 Probes Master Mix (Roche Diagnostics, Mannheim, Germany), Rab25 (Intergrated DNA Technologies, Inc., Toronto, ON, Canada) and G6PD gene-specific primers (Roche Diagnostics), and Universal Probe Library hydrolysis probes (Roche Diagnostics) labeled with 6-Carboxyflourescein or VIC at the 5'-end, and with Non-flourescent Dark Quencer dye or HEX at the 3'-end, for Rab25 and G6PD, respectively. The following primer sequences were used: Forward, 5'-GCTGCTGTCAAGGCTCAGAT-3' and reverse, 5'-CCCACTGCACCACGATAGTA-3' for Rab25; forward, 5'-GAGCCAGATGCACTTCGTG-3' and reverse, 5'-GGGCTTCTCCAGCTCAATC-3' for G6PD. The target and reference genes were co-amplified in the same reaction. Amplification was performed at $95^{\circ} \mathrm{C}$ for $20 \mathrm{sec}, 60^{\circ} \mathrm{C}$ for $15 \mathrm{sec}$ and $72^{\circ} \mathrm{C}$ for $20 \mathrm{sec}$ for 45 cycles, with an initial denaturation at $95^{\circ} \mathrm{C}$ for $10 \mathrm{~min}$, using the Light Cycler $480 \mathrm{II}$ platform (Roche Diagnostics). Changes in the expression levels were calculated using the $2^{-\Delta \Delta \mathrm{Ct}}$ method (33).

Western blotting. Tissue samples were rinsed with ice-cold phosphate-buffered saline and homogenized in a protease inhibitor (PMSF), containing protein extraction buffer (1X lysis buffer, $1 \mathrm{X}$ TBS, $1 \%$ Nonidet P-40, 0.5\% sodium deoxycholate, $0.1 \%$ SDS, $0.004 \%$ sodium azide), in order to obtain whole cell lysate. Equal quantities of protein $(30 \mu \mathrm{g})$ from each sample were loaded onto $12 \%$ SDS-PAGE gels and electrophoresed at $165 \mathrm{~V}$ for $50 \mathrm{~min}$ at room temperature. Resolved protein samples were transferred to nitrocellulose membranes (Invitrogen Life Technologies, Carlsbad, CA, USA) using the iBlot ${ }^{\circledR}$ Dry Blotting system (Invitrogen Life Technologies) for western blot analysis. Following transfer, immunoblots were blocked overnight in 5\% non-fat dry milk (w/v) in Tris-buffered saline (TBS), containing $20 \mathrm{mM}$ Tris- $\mathrm{HCl}$ (pH 7.6), $150 \mathrm{mM} \mathrm{NaCl}$ and $0.1 \%$ Tween-20, and probed with the mouse anti-Rab25 monoclonal antibody, clone 12C3 (cat no. 05-1574; EMD Millipore, Billerica, MA, USA), which was diluted to $1 / 1,000$ with $1 \%$ non-fat dry milk in TBS with Tween-20 (TBST; Santa Cruz Biotechnology, Inc., Dallas, TX, USA). In order to measure Akt1 or phosphorylated Akt1 protein levels, the blots were incubated with primary monoclonal mouse Akt1 (B1; cat. no. sc-5298; 1/2,000 dilution; Santa Cruz Biotechnology, Inc.) and mouse p-Akt1 (5.Ser 473; cat no. sc-293125; 1/2,000 dilution; Santa Cruz Biotechnology, Inc.) antibodies for $90 \mathrm{~min}$ at room temperature, respectively. The blots were washed with TBST three times and the protein content was visualized using HRP-conjugated goat anti-mouse secondary antibodies for Akt1 and p-Akt-1 (cat. no. sc-2005; 1/1,000 dilution; Santa Cruz Biotechnology, Inc.) and Rab25 (cat. no. AP181P; 1/1,000 dilution; EMD Millipore) for $60 \mathrm{~min}$ at room temperature. The protein bands were detected by the Western Lightening Chemiluminescence reagent (Invitrogen Life Technologies). The intensity of luminescence was quantified using the ImageJ 1.47 bundle with the Java analyzing program (http://rsb.info.nih.gov/ij/download.html).

Statistical analysis. The $\chi^{2}$ test was performed to analyze the differences in expression levels between tumor and normal tissues. The correlation between expression levels and clinicopathological parameters was analyzed using the Spearman's Rho test. Statistical analysis was performed using IBM SPSS software (version 20.0.0; IBM SPSS, Armonk, NY, USA). For all statistical tests, $\mathrm{P}<0.05$ was considered to indicate a statistically significant difference. 
Table I. Association of Rab25 protein and mRNA expression with clinicopathological characteristics.

\begin{tabular}{|c|c|c|c|c|c|c|c|c|}
\hline \multirow[b]{2}{*}{ Characteristic } & \multirow[b]{2}{*}{ Patients, n (\%) } & \multicolumn{2}{|c|}{ Rab25 protein, n (\%) } & \multirow[b]{2}{*}{ P-value } & \multicolumn{3}{|c|}{ Rab25 mRNA, n (\%) } & \multirow[b]{2}{*}{ P-value } \\
\hline & & Decreased & Increased & & Decreased & No change & Increased & \\
\hline Location & & & & 0.492 & & & & 0.976 \\
\hline Larynx & $59(77.6)$ & $23(52.3)$ & $21(47.7)$ & & $39(66.1)$ & $3(5.1)$ & $17(28.8)$ & \\
\hline Oral cavity & $9(11.8)$ & $4(57.1)$ & $3(42.9)$ & & $6(66.7)$ & - & $3(33.3)$ & \\
\hline Parotitis & $5(6.6)$ & $3(75.0)$ & $1(25.0)$ & & $4(80.0)$ & - & $1(20.0)$ & \\
\hline Paranasal sinus & $3(3.9)$ & $2(100.0)$ & $0(0.0)$ & & $2(66.7)$ & - & $1(33.3)$ & \\
\hline Stage & & & & 0.547 & & & & 0.411 \\
\hline 1 & $2(2.6)$ & - & - & & $1(50.0)$ & - & $1(50.0)$ & \\
\hline 2 & $27(27.6)$ & $12(66.7)$ & $6(33.3)$ & & $14(51.9)$ & $2(7.4)$ & $4(40.7)$ & \\
\hline 3 & $21(35.5)$ & $9(50.0)$ & $9(50.0)$ & & $17(63.0)$ & - & $10(37.0)$ & \\
\hline 4 & $26(34.2)$ & $11(52.4)$ & $10(47.6)$ & & $19(73.1)$ & $1(3.8)$ & $6(23.1)$ & \\
\hline Histology & & & & 0.384 & & & & 0.692 \\
\hline $\mathrm{SCC}$ & $67(88.2)$ & $27(54.0)$ & $23(46.0)$ & & $44(65.7)$ & $3(4.5)$ & $20(29.8)$ & \\
\hline Other & $9(11.8)$ & $5(71.4)$ & $2(28.6)$ & & $7(57.1)$ & - & $2(42.9)$ & \\
\hline Local invasion & & & & 0.126 & & & & 0.532 \\
\hline Negative & $18(23.7)$ & $7(77.8)$ & $2(22.2)$ & & $11(61.1)$ & $1(5.6)$ & $6(33.3)$ & \\
\hline Positive & $55(72.4)$ & $22(50.0)$ & $22(50.0)$ & & $40(72.7)$ & $1(1.8)$ & $14(25.5)$ & \\
\hline Age, years & & & & 0.080 & & & & 0.850 \\
\hline$\geq 60$ & $36(47.4)$ & $13(44.8)$ & $16(55.2)$ & & $25(69.4)$ & $1(2.8)$ & $10(27.8)$ & \\
\hline$<60$ & $40(52.6)$ & $19(67.9)$ & $9(32.1)$ & & $26(65.0)$ & $2(5.0)$ & $12(30.0)$ & \\
\hline Gender & & & & 0.450 & & & & 0.692 \\
\hline Male & $67(88.2)$ & $29(58.0)$ & $21(42.0)$ & & $44(65.7)$ & $3(4.5)$ & $20(29.8)$ & \\
\hline Female & $9(11.8)$ & $3(42.9)$ & $4(57.1)$ & & 7 (77.8) & & $2(22.2)$ & \\
\hline
\end{tabular}

Rab-25, RAS-associated binding protein 25; SCC, squamous cell carcinoma; n, number.

Table II. Median expression of Rab25 mRNA expression in tumor and normal tissues.

\begin{tabular}{lcccc}
\hline Tissue & Rab25 Ct (median) & G6PD Ct (median) & $\Delta C t$ & $\Delta \Delta C t$ \\
\hline HNSCC & 29.45 & 26.96 & 2.50 & 1.77 \\
Normal & 28.77 & 28.03 & 0.73 & 0.29 \\
\hline
\end{tabular}

Rab25, RAS-associated binding protein 25; G6PD, glucose-6 phosphate dehydrogenase; HNSCC, head and neck squamous cell carcinoma; $\mathrm{Ct}$, cycling threshold.

\section{Results}

Rab25 mRNA expression is decreased in HNSCC, although its expression is not correlated with tumor invasion. Rab25 mRNA expression was analyzed using RT-qPCR in samples from patients with HNSCC and compared with matched adjacent non-malignant tissues. Complete loss of Rab25 mRNA expression was observed in 9 out of $76(11.8 \%)$ tumor samples. When the mean $\Delta \mathrm{Ct}$ level of the remaining 67 samples was calculated, a 3.44-fold decrease in Rab25 expression was observed in the tumor samples compared with that in the non-malignant tissue samples, following normalization to G6PD (Table II). Decreased Rab25 expression was

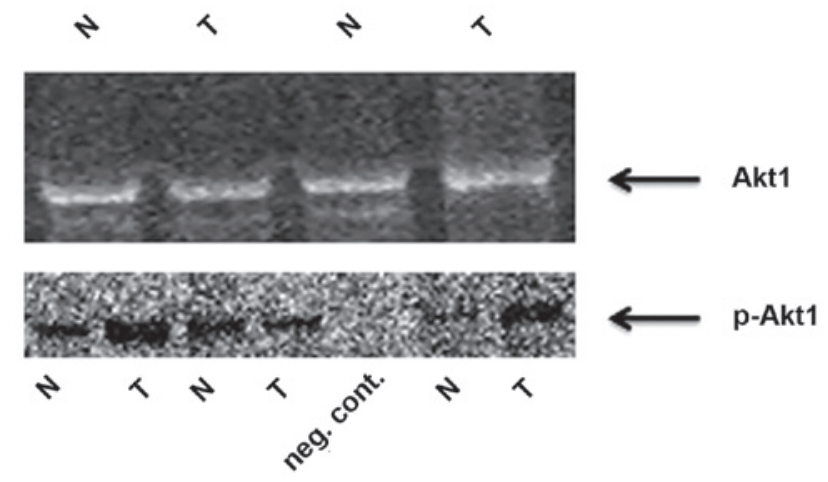

Figure 1. Representative western blot of tumor and matched non-tumor tissue samples. N, non-tumor; T, tumor; p-Akt1, phosphorylated-Akt1. 
observed in $69.1 \%$ of cases. The majority of patients were male (88.2\%), and had laryngeal cancer (77.6\%) and SCC $(88.2 \%)$. Fifty-five of the patients had evidence of vascular (26\%), lymphatic (57.5\%), perineural (39.7\%) and/or cartilage invasion (43.7\%). However, when the association between Rab25 expression and invasion was analyzed, no significant correlation was detected between Rab25 expression and tumor invasion $(\mathrm{P}=0.529)$. Similarly, no significant correlation was identified between Rab25 expression and other clinicopathological variables (Table I). None of the patients had evidence of metastasis to any other site.

Rab25 protein is downregulated and p-Aktl is upregulated in HNSCC. The Rab25 protein was also downregulated in $56.1 \%$ of tumor tissues and this downregulation at the protein level was positively correlated with Rab25 mRNA expression. By contrast, p-Akt1 expression was increased in tumor tissues, compared with that in non-cancerous tissues (Fig. 1), and this upregulation in p-Akt1 levels was inversely correlated with Rab25 protein expression $(\mathrm{P}=0.032)$.

\section{Discussion}

Malfunction of the vesicular traffic system is an abnormal biological behavior that occurs in cancer cells. It has been shown that the Ras superfamily proteins regulate various steps of vesicular trafficking in eukaryotic cells (34). Rab25, a member of the Ras superfamily, has been demonstrated to be involved in cell proliferation and in protection of cells from apoptosis $(22,23-35)$. Initially, Rab25 was hypothesized to be associated with epithelial cancer, following reports that Rab25 is one of $6 \mathrm{Rab}$ proteins that are upregulated in hepatocellular carcinoma (17). Subsequently, Rab25 was identified as a driver of the 1q22 amplicon, which is frequently amplified in ovarian cancer (22-35). In a separate study, Rab25 expression was also shown to be correlated with histological grade and it was defined as an androgen response gene in ovarian cancer (36). Increased expression of Rab25 was observed in breast, ovarian and bladder cancer, and was associated with aggressive tumor features $(22,23,28)$. By contrast, a tumor suppressor role for Rab25 has also been demonstrated in colon cancer, triple-negative breast cancer and esophageal SCC (ESCC) $(31,37,38)$. These results indicate that changes in the expression of Rab25 may depend on the cell type and, therefore, the mechanism underlying the effect of this protein on cancer progression may be tumor-type specific. Although data on the involvement of the Rab25 in proliferation, survival, apoptotic signaling, angiogenesis, invasion and metastasis are available, to the best of our knowledge there is no evidence of the precise molecular mechanisms underlying its effects. One study has suggested that it affects TIMP1-associated tumor growth and angiogenesis (39), while another reported that Rab25 is a regulator of cellular bioenergetics in cancer (40). Caswell et al (41) hypothesized that Rab25 promotes $\alpha 5 \beta 1$ integrin-containing vesicle trafficking to the membrane of pseudopodia tips, thereby facilitating cell invasion in ovarian cancer. A further study demonstrated that overexpression of Rab25 in rat intestinal cells may lead to microtubule-dependent transformation in vitro and tumor formation in vivo (42). Multiple studies have implicated Rab25 in invasion and cell migration. However, the results have been contradictory, with certain studies demonstrating an association between upregulation of Rab25 gene expression, and metastasis and invasion, while other studies have produced the opposite result. In order to evaluate the mechanisms underlying its effects on invasion and metastasis, functional experiments have been performed. Zhang et al (25) reported that Rab25 contributes to metastasis through induction of epithelial mesenchymal transition and activation of the Akt/GSK-3 $3 /$ Snail pathway in bladder cancer. Increased Akt phosphorylation has also been associated with overexpression of Rab25 in aggressive breast and ovarian cancer in vitro (22). Tong et al (37) reported that Rab25 mediates tumorigenicity, metastasis and angiogenesis in ESCC, via deregulation of FAK-Raf-MEK1/2-ERK signaling. By contrast, Amornphimolthan et al (32) demonstrated that Rab25 regulated cell migration and metastasis by modulating the epidermal growth factor receptor-mediated pathway in oral cancer. Until recently there were no reports on the expression level of Rab25 in HNSCC. However, in 2013, Amornphimolthan et al (32) reported that Rab25 functions as a tumor suppressor in HNSCC. In their study, the expression of Rab25 in vitro and in vivo was measured, in association with invasion and metastasis in HNSCC, and it was concluded that downregulation of Rab25 is associated with invasion and metastasis in this disease. However, to date that have been no studies investigating the role of Rab25 in human HNSCC. Therefore, in order to examine the effect of Rab25 in HNSCC the present study was conducted using tissue samples from patients with this disease. In accordance with the results reported by Amornphimolthan et al (32), the present study demonstrated that Rab25 was significantly downregulated in $69.1 \%$ and $56.1 \%$ of the HNSCC tumor tissue samples at the mRNA and protein levels, respectively. However, in contrast to the results of the study by Amornphimolthan et al (32), Rab25 downregulation was not associated with tumor invasion in the current study $(\mathrm{P}=0.529)$. In accordance with in vitro studies reported by Zhang et al (25), downregulation of Rab25 was associated with increased Akt1 phosphorylation, which is an indicator of Akt1 activation. The results of a study by Tang et al (43) suggested a correlation between loss of Rab25 expression, and H-ras or K-ras mutations in breast cancer cell lines. In the present study group all of the patients were K-ras-negative. Therefore, the current results do not support the hypothesis proposed by Tang et al.

The majority of studies published on this subject have been performed using cell lines and animal models to investigate gene expression profiles. The regulation of gene expression in vivo or under physiological conditions may be distinct from that observed in vitro. To the best of our knowledge, this is the first study to investigate Rab25 expression levels in human HNSCC tissue samples. The results indicate that Rab25 functions as a tumor suppressor in HNSCC. However, its downregulation was not found to be associated with lymphatic, vascular or cartilage invasion. All patients in the current study population were metastasis-free, and in the absence of distant metastasis, Rab25 was shown to be downregulated at the mRNA and protein level in HNSCC tumor tissues. This downregulation was associated with an increase in Akt1 phosphorylation. The current results indicate that Rab25 primarily functions as a tumor suppressor in HNSCC and exerts its effect by preventing Akt1 phosphorylation, which is a molecule that is known to activate the mTOR pathway. In conclusion, Rab25 
may have exert tumor suppressor or oncogenic effects during tumor progression, depending on the cell type; suppressing tumor progression by blocking Aktl phosphorylation in particular types of tumor and promoting progression by phosphorylating Akt1 in others. However, further functional studies are required to elucidate the precise mechanisms underlying these effects.

\section{Acknowledgements}

This study was supported by the Research Fund of Istanbul University (grant nos. 13732 and 34503).

\section{References}

1. Warnakulasuriya S: Global epidemiology of oral and oropharyngeal cancer. Oral Oncol 45: 309-316, 2009.

2. Hashibe M, Brennan P, Chuang SC, et al: Interaction between tobacco and alcohol use and the risk of head and neck cancer: Pooled analysis in the International Head and Neck Cancer Epidemiology Consortium. Cancer Epidemiol Biomarkers Prev 18: 541-550, 2009.

3. Howlader N, Noone AM, Krapcho M, et al (eds): SEER Cancer Statistics Review, 1975-2010, National Cancer Institute. Bethesda, MD, http://seer.cancer.gov/csr/1975_2010/, based on November 2012 SEER data submission, posted to the SEER web site, April 2013.

4. Báez A: Genetic and enviromental factors in head and neck cancer genesis. J Environ Sci Health C Environ Carcinog Ecotoxicol Rev 26: 174-200, 2008.

5. Gleich LL and Salamone FN: Molecular genetics of head and neck cancer. Cancer Control 9: 369-378, 2002.

6. Demokan S, Suoglu Y, Demir D, et al: Microsatellite instability and methylation of the DNA mismatch repair genes in head and neck cancer. Ann Oncol 17: 995-999, 2006.

7. Pereira-Leal JB and Seabra MC: Evolution of the Rab family of small GTP-binding proteins. J Mol Biol 313: 889-901, 2001.

8. Zerial $\mathrm{M}$ and $\mathrm{McBride} \mathrm{H}$ : Rab proteins as membrane organizers. Nat Rev Mol Cell Biol 2: 107-117, 2001.

9. Deneka M, Neeft M and van der Sluijs P: Regulation of membrane transport by rab GTPases. Crit Rev Biochem Mol Biol 38: 121-142, 2003.

10. Pfeffer S and Aivazian D: Targeting Rab GTPases to distinct membrane compartments. Nat Rev Mol Cell Biol 5: 886-896, 2004.

11. Jordens I, Marsman M, Kuijl C and Neefjes J: Rab proteins, connecting transport and vesicle fusion. Traffic 6: 1070-1077, 2005

12. Schwartz SL, Cao C, Pylypenko O, Rak A and Wandinger-Ness A: Rab GTPases at a glance. J Cell Sci 120: 3905-3910, 2007.

13. Somsel Rodman J and Wandinger-Ness A: Rab GTPases coordinate endocytosis. J Cell Sci 113 Pt 2: 183-92, 2000.

14. Vetter IR and Wittinghofer A: The guanine nucleotide-binding switch in three dimensions. Science 294: 1299-1304, 2001.

15. Goldenring JR, Shen KR, Vaughan HD and Modlin IM Identification of a small GTP-binding protein, Rab25, expressed in the gastrointestinal mucosa, kidney and lung. J Biol Chem 268: 18419-18422, 1993

16. He H, Dai F, Yu L, et al: Identification and characterization of nine novel human small GTPases showing variable expressions in liver cancer tissues. Gene Expr 10: 231-242, 2002.

17. Casanova JE, Wang X, Kumar R, et al: Association of Rab25 and Rab11a with the apical recycling system of polarized Madin-Darby canine kidney cells. Mol Biol Cell 10: 47-61, 1999.

18. Wang X, Kumar R, Navarre J, et al: Regulation of vesicle trafficking in madin-darby canine kidney cells by Rab1la and Rab25. J Biol Chem 275: 29138-29146, 2000.

19. Agarwal R, Jurisica I, Mills GB and Cheng KW: The emerging role of the RAB25 small GTPase in cancer. Traffic 10: 1561-1568, 2009.

20. Cheng KW, Lahad JP, Gray JW and Mills GB: Emerging role of RAB GTPases in cancer and human disease. Cancer Res 65: 2516-2519, 2005
21. Mitra S, Cheng KW and Mills GB: Rab25 in cancer: A brief update. Biochem Soc Trans 40: 1404-1408, 2012.

22. Cheng KW, Lahad JP, Kuo WL, et al: The RAB25 small GTPase determines aggressiveness of ovarian and breast cancers. Nat Med 10: 1251-1256, 2004.

23. Fan Y, Xin XY, Chen BL and Ma X: Knockdown of RAB25 expression by RNAi inhibits growth of human epithelial ovarian cancer cells in vitro and in vivo. Pathology 38: 561-567, 2006.

24. Liu Y, Tao X, Jia L, et al: Knockdown of RAB25 promotes autophagy and inhibits cell growth in ovarian cancer cells. Mol Med Rep 6: 1006-1012, 2012.

25. Zhang J, Wei J, Lu J, et al: Overexpression of Rab25 contributes to metastasis of bladder cancer through induction of epithelial-mesenchymal transition and activation of Akt/GSK-3ß/Snail signaling. Carcinogenesis 34: 2401-2408, 2013.

26. Calvo A, Xiao N, Kang J, et al: Alterations in gene expression profiles during prostate cancer progression: Functional correlations to tumorigenicity and down-regulation of selenoprotein-P in mouse and human tumors. Cancer Res 62: 5325-5335, 2002.

27. Wang W, Goswami S, Lapidus K, et al: Identification and testing of a gene expression signature of invasive carcinoma cells within primary mammary tumors. Cancer Res 64: 8585-8594, 2004.

28. Mor O, Nativ O, Stein A, et al: Molecular analysis of transitional cell carcinoma using cDNA microarray. Oncogene 22: 7702-7710, 2003.

29. Natrajan R, Williams RD, Hing SN, et al: Array CGH profiling of favourable histology Wilms tumours reveals novel gains and losses associated with relapse. J Pathol 210: 49-58, 2006.

30. Cheng JM, Ding M, Aribi A, et al: Loss of RAB25 expression in breast cancer. Int J Cancer 118: 2957-2964, 2006.

31. Goldenring JR and Nam KT: Rab25 as a tumour suppressor in colon carcinogenesis. Br J Cancer 104: 33-36, 2011.

32. Amornphimoltham $\mathrm{P}$, Rechache $\mathrm{K}$, Thompson J, et al: Rab25 regulates invasion and metastasis in head and neck cancer. Clin Cancer Res 19: 1375-1388, 2013.

33. Schmittgen TD and Livak KJ: Analyzing real-time PCR data by the comparative C(T) method. Nat Protoc 3: 1101-1108, 2008.

34. Grosshans BL, Ortiz D and Novick P: Rabs and their effectors: Achieving specificity in membrane traffic. Proc Natl Acad Sci USA 103: 11821-11827, 2006.

35. Cheng KW, Lu Y and Mills GB: Assay of Rab25 function in ovarian and breast cancers. Methods Enzymol 403: 202-215, 2005.

36. Sheach LA, Adeney EM, Kucukmetin A, et al: Androgen-related expression of G-proteins in ovarian cancer. Br J Cancer 101: 498-503, 2009.

37. Tong M, Chan KW, Bao JY, et al: Rab25 is a tumor suppressor gene with antiangiogenic and anti-invasive activities in esophageal squamous cell carcinoma. Cancer Res 72: 6024-6035, 2012.

38. Cheng JM, Volk L, Janaki DK, Vyakaranam S, Ran S and Rao KA: Tumor suppressor function of Rab25 in triple-negative breast cancer. Int J Cancer 126: 2799-2812, 2010.

39. Bigelow RL, Williams BJ, Carroll JL, et al: TIMP-1 overexpression promotes tumorigenesis of MDA-MB-231 breast cancer cells and alters expression of a subset of cancer promoting genes in vivo distinct from those observed in vitro. Breast Cancer Res Treat 117: 31-44, 2009.

40. Cheng KW, Agarwal R, Mitra S, et al: Rab25 increases cellular ATP and glycogen stores protecting cancer cells from bioenergetic stress. EMBO Mol Med 4: 125-141, 2012.

41. Caswell PT, Spence HJ, Parsons M, White DP, Clark K, Cheng KW, Mills GB, Humphries MJ, Messent AJ, Anderson KI, et al: Rab25 associates with alpha5betal integrin to promote invasive migration in 3D microenvironments. Dev Cell 13: 496-510, 2007.

42. Lapierre LA, Caldwell CM, Higginbotham JN, Avant KM, Hall J, Beauchamp RD and Goldenring JR: Transformation of rat intestinal epithelial cells by overexpression of Rab25 is microtubule dependent. Cytoskeleton (Hoboken) 68: 97-111, 2011.

43. Tang BL: Is Rab25 a tumor promoter or suppressor-context dependency on RCP status? Tumor Biol 31: 359-361, 2010. 